\title{
Dynamic Model Identification for Insect Electroantennogram with Printed Electrode
}

\author{
Naoki Yamada, ${ }^{1}$ Hirono Ohashi, ${ }^{1}$ Takuya Umedachi, ${ }^{2}$ \\ Masahiro Shimizu, ${ }^{1}$ Koh Hosoda, ${ }^{1}$ and Shunsuke Shigaki ${ }^{*}$ \\ ${ }^{1}$ Department of System Innovation, Osaka University, 1-2 Machikaneyama-cho, Toyonaka, Osaka 560-0043, Japan \\ ${ }^{2}$ Faculty of Textile Science and Technology, Shinshu University, 3-15-1 Tokida, Ueda, Nagano 386-8567, Japan
}

(Received September 25, 2020; accepted February 16, 2021; online published February 19, 2021)

Keywords: dynamic model identification, electroantennogram, printed electronics

The purpose of this study is to establish a method by which anyone can easily use an insect antenna as a robot olfactory sensor. Research has been conducted to use an insect antenna as a robot olfactory sensor because insect antennae are much more selective and sensitive than artificial odor sensors. To use an antenna as a robot olfactory sensor, a robot was controlled on the basis of the change in the electroantennogram (EAG) response to odor. However, EAG measurements were performed using microelectrodes or glass electrodes. Hence, it is difficult for engineering researchers and others who are not familiar with microscopic work to easily use an antenna as an odor sensor. For that reason, in this study, we designed a printed electrode using printed electronics (PE) technology that can be used for EAG measurement even for different morphologies of antennae. To detect the presence or absence of odor from the EAG response, we used a dynamic model to eliminate the effects of hum noise and drift associated with the measurement of biological signals. As a result, we proposed a method to make insect antennae easily usable for olfaction for an autonomous robot.

\section{Introduction}

Automated production and big-data-driven artificial intelligence methods have dramatically increased computational power. Consequently, artificial systems have achieved excellent performance for operation in familiar environments, ${ }^{(1,2)}$ but their ability to adapt to new environments remains limited. ${ }^{(3)}$ A common task is searching for sources using olfactory sensing, for which dogs are currently used. Unlike sound and/or light, odor molecules in the atmosphere are present at extremely low spatiotemporal concentrations, which nontrivially depend on wind and/or temperature; this complicates the task of autonomous robotic sensing. ${ }^{(4)}$ However, this ability has significant social and academic value, because it can be used in autonomous robots for life-saving tasks and for identifying the sources of toxic substance leaks at disaster sites, where it may be difficult to conduct vision-assisted search.

${ }^{*}$ Corresponding author: e-mail: shigaki@arl.sys.es.osaka-u.ac.jp https://doi.org/10.18494/SAM.2021.3116 
Animals skillfully use odor-related information to identify feeding sites, find mates, and distinguish between enemies and allies. In particular, insects can identify odor types and find odor sources in real time, although their computing power is arguably much weaker than that of the latest central processing units (CPUs). Accordingly, many researchers have modeled the search behavior of insects and implemented it in autonomous robots. ${ }^{(5)}$ However, even in a controlled environment such as a laboratory, autonomous robots have not been able to achieve the same search performance as insects. ${ }^{(6)}$ One of the factors that undermines the olfactory performance of autonomous robots is the difficulty of obtaining and equipping artificial odor sensors that would be highly sensitive and highly selective for odorants. The time resolutions of the electroantennogram (EAG) responses of a typical insect and a commonly available odor sensor differ approximately tenfold, resulting in different search performances. ${ }^{(6)}$ Thus, it is important to improve the time resolution of artificial odor sensors, but it is difficult to improve their response performance because artificial odor sensors volatilize chemicals attached to them using a heater and detect the presence or absence of odor molecules on the basis of the resistance change at that time. ${ }^{(7)}$ Therefore, research has focused on using insects' antennae for robot olfaction. ${ }^{(8,9)}$ The key to mounting and using insect antennae on a robot is (1) stable acquisition of the EAG and (2) robust signal processing with respect to noise and/or drift. Previously, ${ }^{(8,9)}$ problem (1) was solved by inserting minute wire electrodes or glass electrodes into both ends of the antennae. However, this method requires significant electrophysiology expertise, which precludes engineers from easily employing insects' antennae for robot olfaction. To solve this problem, we use printed electronics (PE) technology, which has high flexibility and elasticity, and allows us to easily measure EAG responses. ${ }^{(10,11)} \mathrm{PE}$ is used for manufacturing electric circuits and electrodes, and is very accessible because it can be realized using a home-use inkjet printer. In this study, two types of antennae with different morphologies were mounted on printed electrodes designed using PE technology, and the EAG responses were measured.

When measuring EAG responses in a Faraday cage, problem (2) does not occur, but when measuring EAG responses for a robot that operates in a state disconnected from the ground (GND), problem (2) is inevitable. To deal with this problem, it is common to apply analog and digital filters to EAG signals, ${ }^{(8)}$ but this solution is not ideal. Thus, a previous study used a neuromorphic detector based on the Hodgkin-Huxley model for odor patch detection. ${ }^{(9)}$ However, this model has many free parameters, and the method for determining the values of these parameters is quite complicated. ${ }^{(12)}$ In addition, free parameters that require complicated calculations must be adjusted according to the type of antenna. Another problem is that the results may vary slightly depending on the numerical method used for solving the HodgkinHuxley model itself. We overcome this problem by using an autoregressive with exogenous (ARX) model, which has been used as a dynamic filter for artificial odor sensors, ${ }^{(13)}$ for the EAG, because the EAG waveforms are similar to those of artificial odor sensors, although their temporal resolutions are different. Therefore, we applied the ARX model to the EAG waveforms measured by the PE, and performed an inverse model estimation to detect the presence of odor molecules in the atmosphere. Here, we describe a method for the easy use of EAGs in robotic olfaction, and demonstrate its better performance compared with previous methods. 


\section{Problem Statement}

Our goal is to sensorize an insect antenna to demonstrate the utility of the PE technology. For convenience, we refer to such a sensor as a bio-odor sensor.

When odorant molecules adhere to the insect antenna, a bioelectric potential (EAG) of several $\mathrm{mV}$ is generated by the antenna. This EAG signal is transmitted to the insect's brain, consequently affecting the insect's behavior. The EAG concept was proposed by Schneider ${ }^{(14)}$ in the mid-1950s. The EAG can be measured by inserting electrodes at both ends of the insect antenna while it is separated from the insect body. This approach significantly contributed to biological and engineering fields, because it not only allows the sensing function of the insect antenna to be elucidated, but also suggests that an antenna can be used as an odor sensor; this second point is the focus of the present study. Thus, we addressed the following issues:

- Fabrication of electrodes that can be sensorized even with antennae of different structures.

- Modeling of bio-odor sensors.

- Robotic implementation of bio-odor sensors.

Insects have different antenna morphologies depending on the species. ${ }^{(15)}$ Here, we experimentally show that different morphologies can be sensorized. We employed the antennae of a silkmoth (Bombyx mori) and a bumblebee (Bombus ignitus) as those with different morphologies. The antenna of a silkmoth has a comblike morphology with many flagella, whereas that of a bumblebee has a rodlike morphology. These antennae were sensorized by electrodes designed using PE technology. The resulting bio-odor sensors were modeled and mounted on autonomous robots to evaluate their functionality as odor sensors.

\section{Sensorization of Insect Antennae Using the PE Method}

\subsection{Design of printed electrode}

Here, we describe the PE-based fabrication of electrodes for EAG measurements. Figure 1 shows the printing of the electrodes and the external dimensions of the electrodes. The electrodes were printed using a commercially available inkjet printer (PX-S160T, EPSON, Japan) filled with silver nanoink (NBSIJ-MU01, Mitsubishi Paper Mills Limited, Japan). A transparent film (NB-TP-3GU100, Mitsubishi Paper Mills Limited, Japan) was set in the printer, and the designed electrodes were printed on the film. Presentation software (PowerPoint,

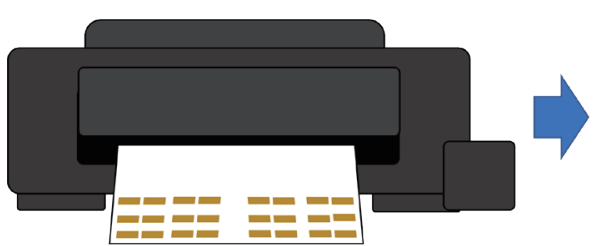

(a)

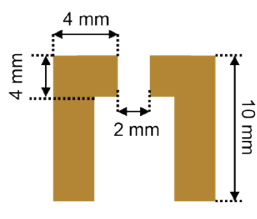

(b)

Fig. 1. (Color online) Procedure for fabricating printed electrodes. (a) Printing of electrodes with an inkjet printer. (b) External dimensions of printed electrode. 
Microsoft, USA) was used to design the printed electrodes. In our previous research, ${ }^{(16)}$ the basic electrical characteristics of this ink and the film composition were comprehensively investigated, and it was found that the longer the side length and the narrower the width, the larger the resistance. This electrical characteristic is the same as that of a general lead wire, and it has been reported that EAGs can be measured even with a general IC pin socket; ${ }^{(17,18)}$ therefore, we assumed that EAGs can be adequately measured with this printed electrode.

As shown in Fig. 1(b), the printed electrode is L-shaped to secure the part on which the antenna is placed and the part connected to an amplifier. The potential difference across the two ends of the antenna was measured by arranging the two L-shapes to face each other. The dimensions of one L-shape were $10 \times 4 \mathrm{~mm}^{2}$ and the distance between the L-shapes was $2 \mathrm{~mm}$.

In this study, the antennae of a silkmoth and a bumblebee, which have completely different morphologies, were considered. As shown in Fig. 2, the antennae were physically excised from the moth and bee, using scissors for dissection. At this time, the antennae tips (approximately $1 \mathrm{~mm}$ ) were cut with scissors to measure the potential difference. After excising the antennae and setting the electrodes, to fix the antennae and improve their connection with the electrodes, we applied a conductive gel (Spectra 360 Electrode Conductive Gel, Parker Laboratories Inc., USA), which is commonly employed for measuring EAGs, ${ }^{(19,20)}$ using pointed tweezers. After installing the antennae, they were connected to the amplifiers and their responses to odor stimuli were measured. The actual EAG responses and modeling are described in the next section.

\subsection{EAG measurements and sensor identification}

Here, we describe the basic response characteristics and modeling of bio-odor sensors. To evaluate the studied bio-odor sensors, an experiment was conducted in which odors were presented to the sensors, and the sensors' outputs with respect to the odor input were evaluated.
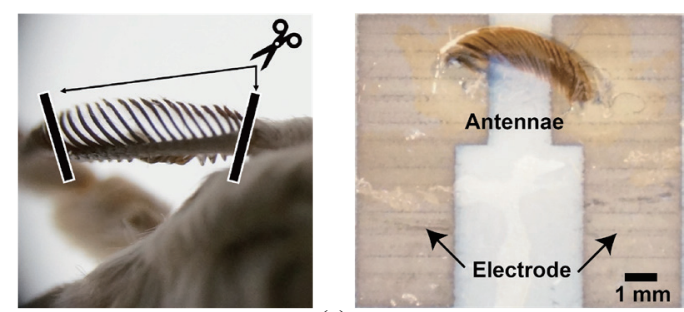

(a)
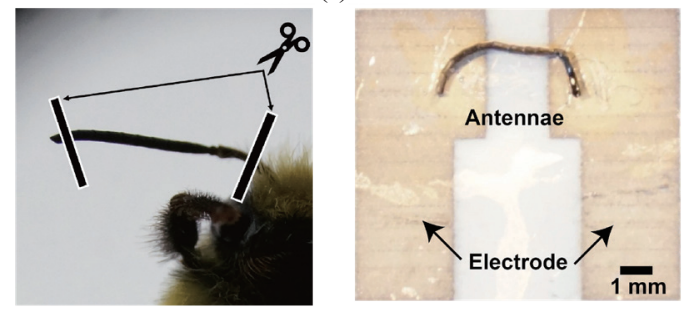

(b)

Fig. 2. (Color online) Isolation of antenna and mounting on the printed electrode. (a) Silkmouth. (b) Bumblebee. 
The odor presentation device ${ }^{(18)}$ used in the experiment consisted of an air compressor (NIP30L, Nihon Denko, Japan), three gas-washing bottles (absorbent cotton, activated carbon, and distilled water), a flow meter (KZ-7002-10A, AZ ONE Corporation, Japan), and a solenoid valve (VT307, SMC Corporation, Japan). The air expelled from the air compressor was passed through absorbent cotton, activated carbon, and distilled water, controlled at a constant flow rate of $2.0 \mathrm{~L} / \mathrm{min}$ by the flowmeter, and presented to the bio-odor sensors. The stimulation timing for the sensors was controlled by changing the state of the solenoid valve. In this experiment, the odors were presented by opening and closing the solenoid valve at a frequency of $0.5 \mathrm{~Hz}$ (open valve duration: $200 \mathrm{~ms}$, closed valve interval: $1800 \mathrm{~ms}$ ), and the sensors' responses were measured and analyzed. The air sent from the solenoid valve passed through the connected glass tube and was presented to the bio-odor sensors. The glass tube contained a filter paper onto which the odorants were dropped, and the odorized air flowed out of the glass tube. When conducting experiments with the silkmoth and bee antennae, a sex-related pheromone (bombykol) and a lemon odor component (citral) were dropped onto the filter paper. Bombykol (1 $\mu \mathrm{g}$ of $1000 \mathrm{ng} / \mu \mathrm{L}$ bombykol, $4.19 \mathrm{~mol})$ and citral $(5 \mu \mathrm{g}$ of $10 \%$ citral, $2.86 \mu \mathrm{mol})$ were deposited on the filter paper. In addition, the glass tube was replaced according to the type of odorous substance. The fixture was made using a three-dimensional (3D) printer, so that the distance between the bio-odor sensor and the discharge port during the experiment was kept constant. The distance between the sensor and the discharge port was $10 \mathrm{~mm}$ for both studied bio-odor sensors. A corresponding printed electrode was connected to an amplifier (AB-651J, Nihon Kohden, Japan) for measurements, because the EAG exhibited a potential change of several $\mathrm{mV}$. The amplifier was equipped with an analog bandpass filter $(0.08-300 \mathrm{~Hz})$. The signal input to the amplifier was recorded using a personal computer (PC) at a sampling rate of $1 \mathrm{kHz}$ using an A/D converter (USB-6215, National Instruments, USA).

In this study, we performed experiments on five antennae. We presented 20 odor stimuli to each antenna.

Figure 3 shows the time series of typical responses of bio-odor sensors. Figures 3(a) and 3(b) respectively show the responses for the silkmoth and bumblebee antennae mounted on the bio-odor sensors. The vertical and horizontal axes in Fig. 3 are the voltage and elapsed

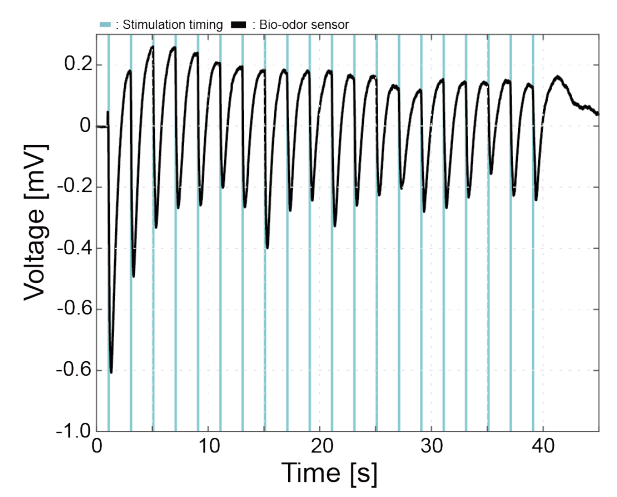

(a)

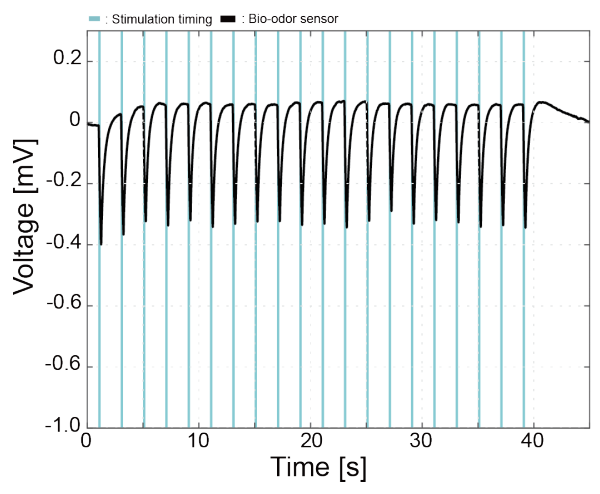

(b)

Fig. 3. (Color online) Typical responses of bio-odor sensors. (a) Silkmoth. (b) Bumblebee. 
time, respectively. The figure suggests a transient inhibitory response to the odor stimulus. Figure 4 shows the sensor responses for both of the studied antennae. Because the absolute voltage amplitude differs depending on the mounting method of the antennae, it is displayed as a normalized value. Figures 4(a) and 4(b) respectively show the responses for the silkmoth and bumblebee antennae mounted on the bio-odor sensors. The red line in Fig. 4 is the average sensor output, while the gray color represents the standard deviation. Clearly, the antennae respond to the presented stimuli for both morphologies. However, it is difficult to use them for stable odor sensing, because the potential change in the output varies with the mounting. Furthermore, it is highly probable that mechanical and electrical noise will interfere with the sensor output for robot-mounted sensors. Therefore, to address this problem, we modeled a bioodor sensor.

We modeled a bio-odor sensor using a basic system identification method because a bioodor sensor is a single-input single-output (SISO) system. We presented M-sequence signal odor stimuli to the modeled bio-odor sensor and identified the system from the odor stimuli input and sensor output relationships. Because the odor frequency in the atmosphere is approximately $0-5 \mathrm{~Hz},{ }^{(21)}$ an M-sequence signal was generated to include frequencies up to $5 \mathrm{~Hz}$. By controlling the opening and closing of the solenoid valve with the generated M-sequence signal, the M-sequence odor stimulus was presented to the sensor. In this study, we modeled a bio-odor sensor using the quadratic ARX inverse model shown in Eqs. (1) and (2).

$$
\begin{gathered}
\hat{u}(k)=b_{0} \dot{y}(k)+b_{1} \dot{y}(k-1)-a_{1} \hat{u}(k-1)-a_{2} \hat{u}(k-2) \\
\hat{u}_{b i n}(k)=\left\{\begin{array}{cc}
\text { Detect } & (\hat{u}(k) \geq \text { Threshold }) \\
\text { Not Detect } & (\hat{u}(k)<\text { Threshold })
\end{array}\right.
\end{gathered}
$$

Here, $\hat{u}(k)$ represents the estimated value of the input at the $k$ th step, $\hat{u}_{b i n}(k)$ is the estimated value of the input at the $k$ th step represented by a binary value, and $\dot{y}(k)[\mathrm{mV} / \mathrm{s}]$ represents the time derivative of the bio-odor sensor output at the $k$ th step. The parameters $a_{1}, a_{2}, b_{0}$, and $b_{1}$

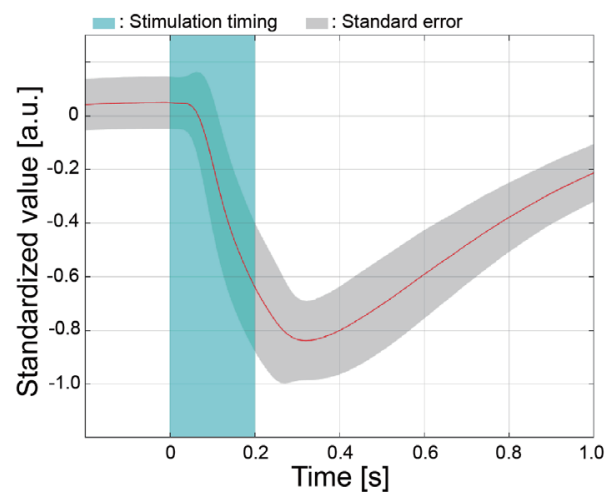

(a)

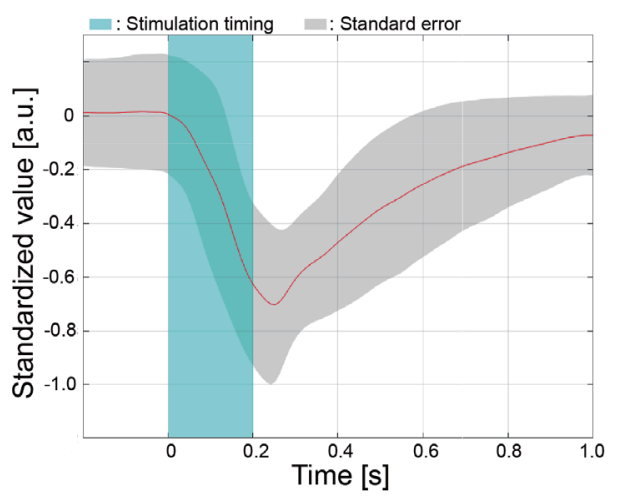

(b)

Fig. 4. (Color online) Average response characteristics of bio-odor sensors. (a) Silkmoth. (b) Bumblebee. 
are the coefficients of the ARX model. The system identification toolbox of MATLAB (2020a, The MathWorks, Inc., MA, USA) was used to select the coefficients of the least squares method for the ARX model. Table 1 lists the coefficients of the estimated ARX model.

Figure 5 shows the results of applying the ARX inverse model to the sensor output. Figures 5(a) and 5(b) respectively show the results for the mounted antennae of the silkmoth and bumblebee. The blue lines represent the times at which the solenoid valve is opened. This corresponds to the time at which the odor stimulus was presented to the bio-odor sensor. The vertical axis represents the voltage ( $\mathrm{mV}$ ) or model output (a.u.), and the horizontal axis represents the experimental time (s). As shown in Fig. 5, after applying the ARX inverse model to the bio-odor sensor output, the model returns an output of the same intensity, even for antennae with different morphologies.

\section{Demonstration of Robot Implementation}

\subsection{Configuration of an autonomous robot}

In the following, we describe the robot configuration used in our experiments. The robot was a nonholonomic moving body with dimensions of $80 \times 50 \times 70 \mathrm{~mm}^{3}$. An odor sensor was attached to the front of the robot and, as shown in Fig. 6(a), the printed electrode with the antenna was sandwiched between a small amplifier and the printing electrode (ISD2PAD, Oisaka Electronic Equipment Ltd., Japan) to measure the EAG [Fig. 6(b)]. The amplification factor was approximately 1000 times, and the incorporated high-pass and low-pass filters were approximately 0.1 and $300 \mathrm{~Hz}$, respectively. ${ }^{(11)}$ The measured EAG was processed on a microcontroller (ESP32, Espressif Systems, China) via an MCP3208 A/D conversion IC (Microchip Technology, USA). The sampling rate was $1 \mathrm{kHz}$. The signal acquisition of the bio-odor sensor

Table 1

Coefficients of the estimated ARX model.

\begin{tabular}{cccc}
\hline$a_{1}$ & $a_{2}$ & $b_{0}$ & $b_{1}$ \\
\hline-0.999 & 0.00133 & -0.0858 & 0.0826 \\
\hline
\end{tabular}

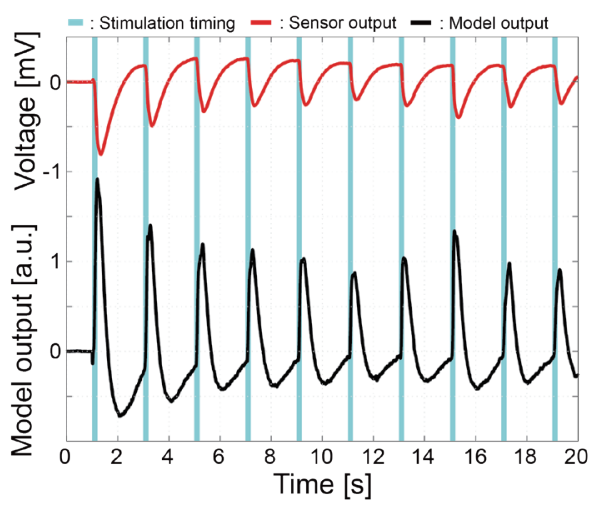

(a)

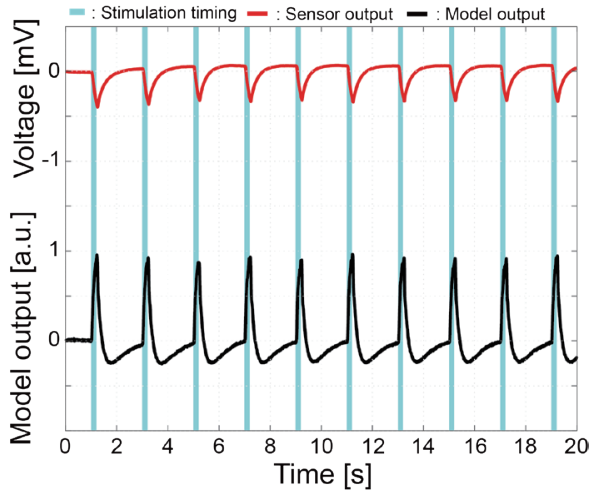

(b)

Fig. 5. (Color online) Response waveform when modeled by the ARX model. (a) Silkmoth. (b) Bumblebee. 


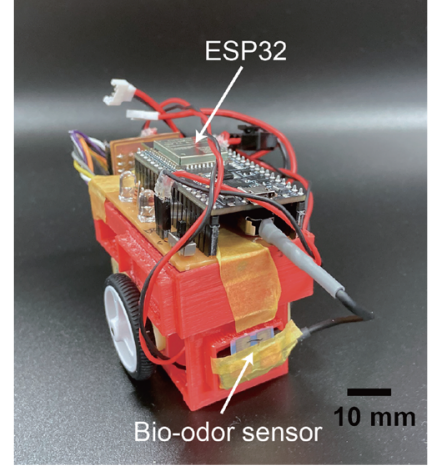

(a) Photo of actual autonomous robot

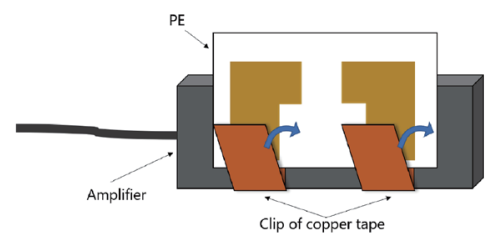

(b) Connection diagram of the amplifier and printed electrode

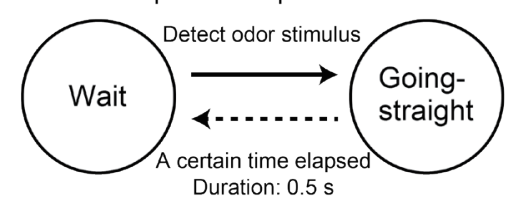

(c) State transition diagram for decision making

Fig. 6. (Color online) Description of the robot configuration.

and the decision-making were performed by the ESP32, and the logged data were transferred to a PC via Bluetooth.

In this experiment, to determine whether the bio-odor sensor can be used for robotic olfaction, the robot moved by performing a simple state transition, shown in Fig. 6(c), while approaching the odor source. The transition to the straightforward state was triggered by the output value of the ARX inverse model, and the transition to the standby state occurred over time. Here, the time elapsed until the standby state was set to $0.5 \mathrm{~s}$. By switching the behavior according to the response of the bio-odor sensor, we investigated whether the odor source could be reached.

\subsection{Experimental conditions}

The mounting experiment on an autonomous robot was carried out in the environment shown in Fig. 7. The distance from the discharge port to the robot was $200 \mathrm{~mm}$. The odor discharge device described in Sect. 3.2 was used. Intermittent odor stimuli at $1 \mathrm{~Hz}$ (open valve, $200 \mathrm{~ms}$; closed valve, $800 \mathrm{~ms}$ ) were emitted from the discharge port. When the antennae of the silkmoth and bumblebee were mounted on the bio-odor sensor, the odorants bombykol and citral were used as odor sources. The trajectory of the robot was recorded using a camera installed in the upper part of the experimental field. The experiment was performed 10 times for each antenna type. To evaluate whether the bio-odor sensor fabricated in this study can be used for robotic olfaction, we analyzed the relationship between the bio-odor sensor readout and the robot trajectory as the robot approached the odor source.

\subsection{Experimental results}

The results representing the relationship between the distance of the robot from the odor source and the EAG response are shown in Fig. 8. The black and red lines at the bottom represent the timing of the odor release from the source and the timing of the odor detection by the robot, respectively. As shown in Fig. 8, the robot approached the odor source based 


\section{(nim}

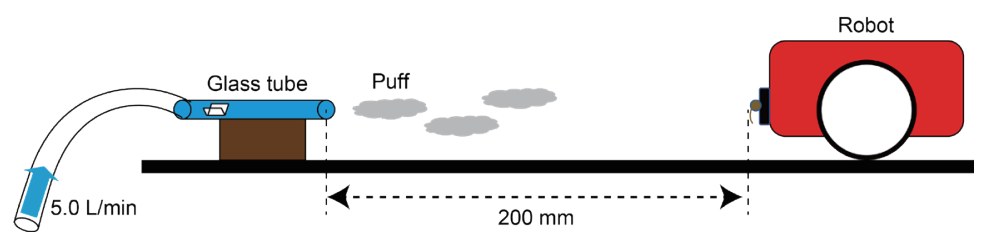

Fig. 7. (Color online) Schematic diagram of the experimental field.

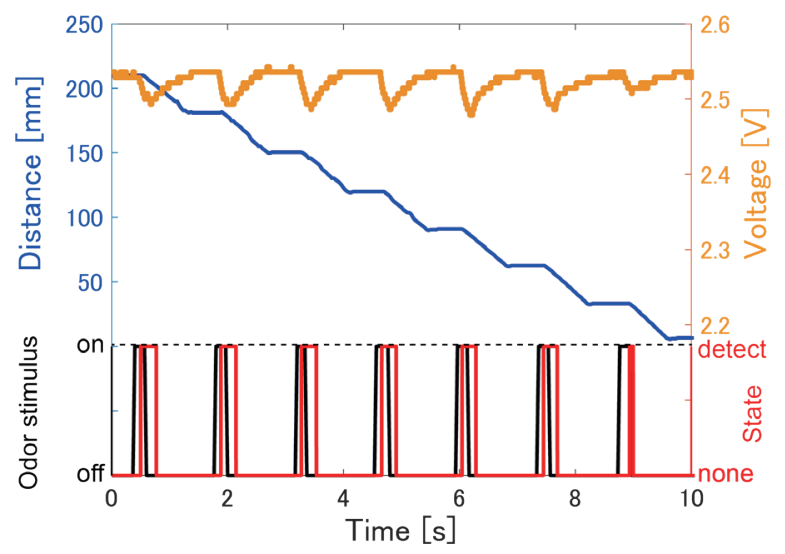

Fig. 8. (Color online) EAG response in relation to distance of robot from odor source. The black and red lines at the bottom represent the timing of the odor emitted from the source and the timing of the odor detection of the robot, respectively.

on the EAG response. It also shows that the robot reached the odor source after detecting approximately seven odor stimuli.

The left side of Fig. 9 shows the success rate of approaching the odor source (see Supplemental Video S1). As is clear from Fig. 9, regardless of the mounted antenna, the robot reliably approached the odor source. As shown in the bar graph in the middle of Fig. 9, the robot reached the odor source after detecting approximately seven stimuli. The detection rate was calculated and evaluated as the number of times the robot successfully detected the odor, because the odor stimuli were presented intermittently by the odor source. The detection rate was defined as

$$
\text { Detection rate }(\%)=\frac{\text { Number of times the robot detected }}{\text { Total number of odor stimuli emitted }} .
$$

From the example in Fig. 8, the detection rate was calculated as $1.0(=7 / 7)$. The detection rate is shown in the bar graph on the right side of Fig. 9, and it was found that both antennae reliably detected the odor stimuli emitted by the odor source. In addition, Fig. 10 shows the change in the sensor output value as the robot approached the odor source. An intermittent odor stimulus of $1 \mathrm{~Hz}$ was presented by the odor source, and the value reported by the bio-odor 

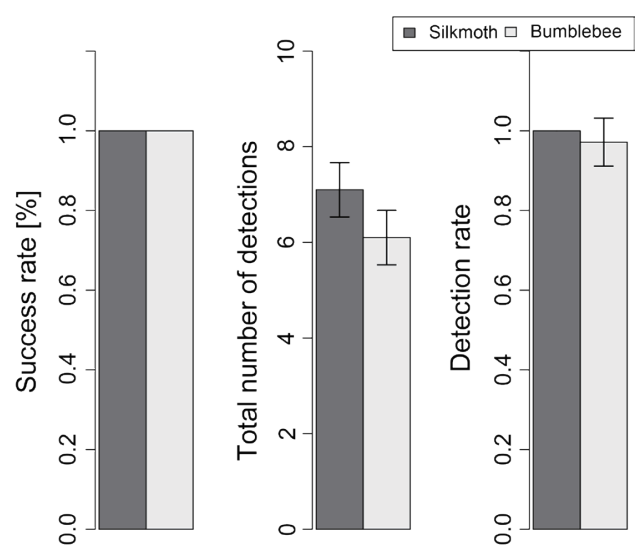

Fig. 9. Results of robot experiments. From left to right, the success rate, total number of detections, and detection rate are shown.
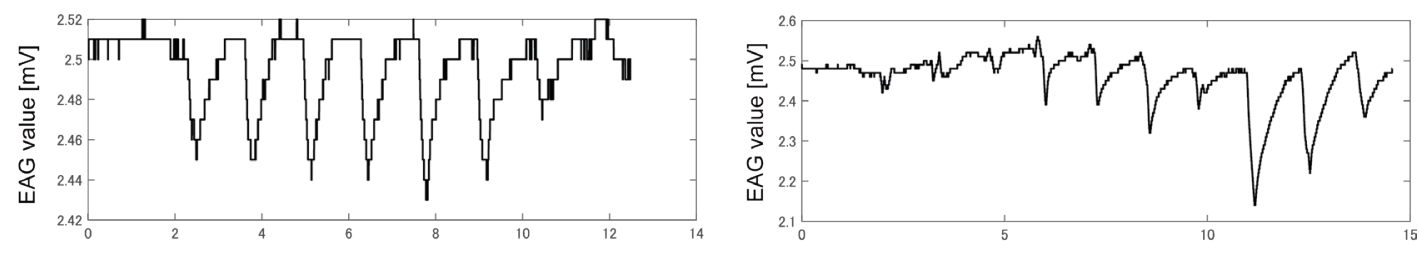

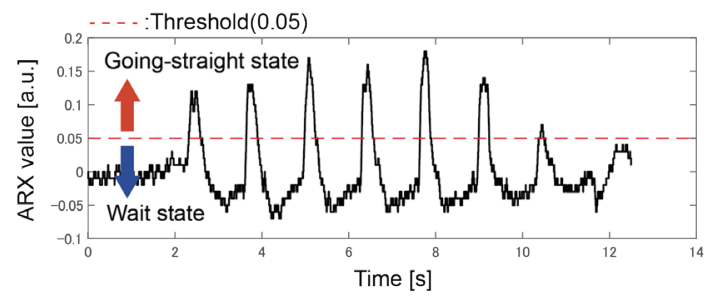

(a)

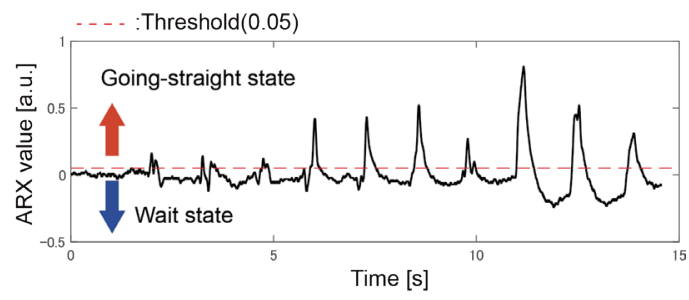

(b)

Fig. 10. (Color online) Typical changes in the odor sensor when approaching the odor source. (a) Silkmoth. (b) Bumblebee.

sensor changed significantly. Furthermore, plotting the value reported by the bio-odor sensor (ARX value) against the distance between the robot and the odor source revealed that the sensor value tended to reach a peak at a certain distance (moth: $80 \mathrm{~mm}$, bee: $40 \mathrm{~mm}$ ) and then decrease.

The Tukey-Kramer test on the ARX values in Fig. 11 suggested a significant difference $(p<0.05)$ between the ARX value at the start of the experiment and the peak ARX value. In the presence of a well-defined gradient (e.g., light or sound), the sensor output should increase monotonically, and it is unlikely that the result shown in Fig. 11 reflects such a situation. This suggests complex patterns associated with the diffusion of odor molecules after they are released from the source.

Overall, the results of this study suggest that the robot can approach the odor source based on the EAG value, and that the bio-odor sensor plays an important role in robotic olfaction. Therefore, we demonstrated that by fabricating printed electrodes using PE technology, insect antenna with various morphologies can be converted into odor sensors. 


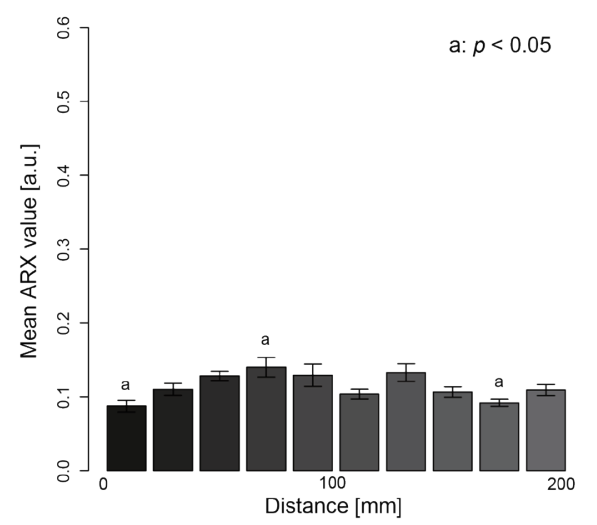

(a)

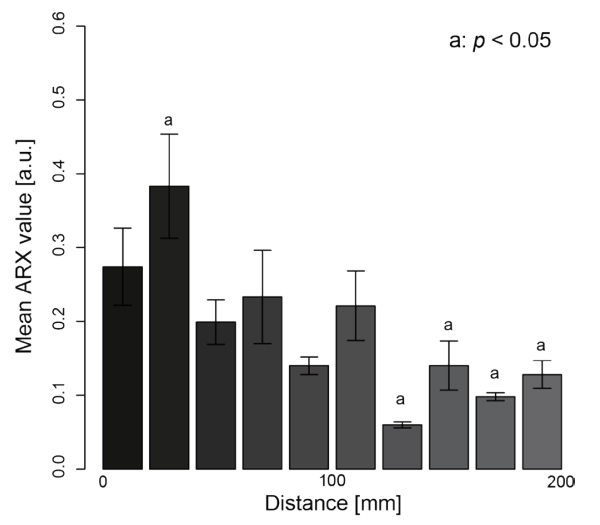

(b)

Fig. 11. Relationship between odor sensor response value and distance from odor source $(n=10)$. (a) Silkmoth. (b) Bumblebee.

\section{Conclusions}

In this study, we established an easy method for measuring EAG waveforms and modeled these waveforms using a general regression model. As a result, EAG waveforms can be measured robustly with respect to the noise and/or drift caused by electrical and mechanical noise, and even engineers without specialized optical equipment (such as microscopes) can use the insects' antennae for robotic olfaction studies.

In this study, we fabricated electrodes for measuring EAGs using PE technology, and converted insect antennae into odor sensors. The electrodes can be easily printed using a household inkjet printer loaded with silver nanoparticles. Different antennae can be considered without changing the electrode, depending on the odorant molecule to be detected, because the electrodes fabricated using PE technology are designed to work with antennae having different morphologies. For convenience, the sensors of the insect antennae fabricated using PE technology are called bio-odor sensors. Because the EAG is a minute signal of several $\mathrm{mV}$, it is expected that mechanical and electrical noise will interfere when such a sensor is installed on a robot. Therefore, the bio-odor sensor was modeled using the ARX model. When the fabricated bio-odor sensor was installed on the robot and approach experiments were conducted, the sensor functioned as an odor sensor for antennae of different morphologies, and the robot was able to reliably reach the odor source. This suggests that the antennae of insects can be easily used for robotic olfaction.

Insect antennae are excellent sensors that respond to various odorants. Therefore, in the future, we aim to construct an "artificial dog" system that will be able to learn and distinguish various odorants using this bio-odor sensor. Furthermore, unlike artificial odor sensors, the EAG response has nonlinearity in that the amplitude attenuates upon exposure to odors in a short period of time (Fig. 5). Such a characteristic cannot be studied with an artificial odor sensor, but it is possible that this characteristic has a role similar to a spatial filter to prevent odor saturation. Hence, we will also investigate how this phenomenon contributes to the function of odor source localization. 


\section{Acknowledgments}

This work was partially supported by the Mitsubishi Foundation and JSPS KAKENHI Grant Numbers JP19K14943, JP18H05467, and JP19K23488. The silkworm strains used in this study were supplied by the National Bio-Resource Project (NBRP) of MEXT, Japan. Moreover, we thank Agrisect Inc. for providing the bumblebees (Bombus ignitus) used in the experiment.

\section{Supplementary Materials}

The following is available online at https://sshigaki.jimdo.com/research/: Video S1: The experiment on approaching odor sources using the robot.

\section{References}

1 M. Dotoli, A. Fay, M. Miśkowicz, and C. Seatzu: Int. J. Product. Res. 57 (2019) 5047. https://doi.org/10.1080/0 $\underline{0207543.2018 .1510558}$

2 A. Shrestha and A. Mahmood: IEEE Access 7 (2019) 53040. https://doi.org/10.1109/ACCESS.2019.2912200

3 C. G. Atkeson, P. B. Benzun, N. Banerjee, D. Berenson, C. P. Bove, X. Cui, D. Mathew, D. Ruixiang, F. Perry, M. Gennert, P, G. Joshua, H. Peng, J. Aaron, K. Joohyung, K. Kevin, L. Lening, L. Chenggang, L. Xianchao, T. Padir, P. Felipe, G. G. Tighe, and X. Xinjilefu: The DARPA Robotics Challenge Finals: Humanoid Robots To The Rescue, Springer Tracts in Advanced Robotics 121 (Springer, 2016) p. 667. https://doi.org/10.1007/978-3319-74666-1 17

4 E. A. Cowen and K. B. Ward: Environ. Fluid Mech. 2 (2002) 1. https://doi.org/10.1023/A:1016247516041

5 X. X. Chen and J. Huang: Rob. Autom. Syst. 112 (2019) 123. https://doi.org/10.1016/j.robot.2018.11.014

6 S. Shigaki, K. Okajima, K. Sanada, and D. Kurabayashi: IEEE Robot. Autom. Lett. 4 (2019) 2847. https://doi. org/10.1109/LRA.2019.2921948

7 J. Burgués and S. Marco: Sensors 18 (2018) 339. https://doi.org/10.3390/s18020339

8 Y. Kuwana, S. Nagasawa, I. Shimoyama, and R. Kanzaki: Biosens. Bioelectron. 14 (1999) 195. https://doi. org/10.1016/S0956-5663(98)00106-7

9 D. Martinez, L. Arhidi, E. Demondion, J. B. Masson, and P. Lucas: J. Vis. Exp. 90 (2014). https://doi. org/10.3791/51704

10 H. Sirringhaus and T. Shimoda: MRS Bulletin 28 (2003) 802. https://doi.org/10.1557/mrs2003.228

11 Y. Kawahara, S. Hodges, B. S. Cook, C. Zhang, and G. D. Abowd: Proc. 2013 ACM Int. Joint Conf. Pervasive and Ubiquitous Computing (2013) 363-372. https://doi.org/10.1145/2493432.2493486

12 J. A. M. Valle and A. L. Madureira: arXiv preprint arXiv (2019). https://arxiv.org/abs/1903.10286v1

13 S. Shigaki, M. R. Fikri, and D. Kurabayashi: Sensors 18 (2018) 3720. https://doi.org/10.3390/s18113720

14 D. Schneider: Zeitschrift für vergleichende Physiologie 40 (1957) 8. https://doi.org/10.1007/BF00298148

15 M. A. Elgar, D. Zhang, Q. Wang, B. Wittwer, T. L. Johnson, C. B. Freelance, and M. Coquilleau: The Yale J. Biol. Med. 91 (2018) 4. https://www.ncbi.nlm.nih.gov/pmc/articles/PMC6302626/

16 N. Yamada, S. Shigaki, M. Shimizu, H. Ohashi, T. Umedachi, T. Ogura, and K. Hosoda: 2020 IEEE/SICE Int. Symp. System Integration (2020). https://doi.org/10.1109/SII46433.2020.9025912

17 B. Lan, R. Kanzaki, and N. Ando: Sensors 19 (2019) 4574. https://doi.org/10.3390/s19204574

18 S. Shigaki, H. Ohashi, T. Sakurai, M. Shimizu, K. Hosoda and D. Kurabayashi: IEEE Sens. Lett. 4 (2020). https://doi.org/10.1109/LSENS.2020.3024606

19 Y. Shiota and T. Sakurai: Odorant Binding Chemosens. Proteins 642 (2020) 325. https://doi.org/10.1016/ bs.mie.2020.05.009

20 B. Antony, J. Johny, and S. A. Aldosari: Front. Physiol. 9 (2018) 1. https://doi.org/10.3389/fphys.2018.00252

21 T. Kikas, H. Ishida, D. R. Webster, and J. Janata: Anal. Chem. 73 (2001) 15. https://doi.org/10.1021/ac0101813 